\title{
Highlights from the VERITAS AGN Observation Program
}

\author{
Wystan Benbow* for the VERITAS Collaboration ${ }^{\dagger}$ \\ Harvard-Smithsonian Center for Astrophysics \\ E-mail: wbenbow@cfa.harvard.edu
}

The VERITAS array of four 12-m imaging atmospheric-Cherenkov telescopes began full-scale operations in 2007, and is one of the world's most sensitive detectors of astrophysical VHE $(\mathrm{E}>100 \mathrm{GeV}) \gamma$-rays. Observations of active galactic nuclei (AGN) are a major focus of the VERITAS Collaboration, and more than 60 AGN, primarily blazars, are known to emit VHE photons. Approximately 3400 hours have been devoted to the VERITAS AGN observation program and roughly 160 AGN are already observed with the array, in most cases with the deepest VHE exposure to date. These observations have resulted in 34 detections, most of which are accompanied by contemporaneous, multi-wavelength observations, enabling a more detailed study of the underlying jet-powered processes. Recent highlights of the VERITAS AGN observation program, and the collaboration's long-term AGN observation strategy, are presented.

The 34th International Cosmic Ray Conference,

30 July- 6 August, 2015

The Hague, The Netherlands

\footnotetext{
*Speaker.

${ }^{\dagger}$ http://veritas.sao.arizona.edu/
} 


\section{Introduction}

AGN emit non-thermal radiation across the entire broadband spectrum and are among the most powerful particle accelerators in the universe. They are the most numerous class of identified VHE $\gamma$-ray emitter and comprise approximately one-third of the VHE sky catalog. As of June 2015, sixty-two AGN are identified as VHE sources. These all belong to the small fraction of AGN which possess jets powered by accretion onto a supermassive black hole (SMBH). It is believed that the $\gamma$-ray emission is produced in these jets, in a compact region near the SMBH event horizon.

Most ( $95 \%$ ) of the VHE $\gamma$-ray emitting AGN are blazars, a class of AGN with jets pointed along the line-of-sight to the observer. The VHE blazar population includes four blazar subclasses: 45 high-frequency-peaked BL Lac objects (HBLs), 7 intermediate-frequency-peaked BL Lac objects (IBLs), 1 low-frequency-peaked BL Lac object (LBL), and 5 flat-spectrum radio quasars (FSRQs), as well as one gravitationally-lensed blazar whose sub-classification is uncertain. Although the attenuation of VHE photons on the extragalactic background light (EBL) leads to an eventual distance horizon, blazars are detected at VHE out to a redshift of $z=0.944$. The only other AGN detected at VHE are three nearby $(z<0.02)$ FR-I radio galaxies.

Empirically, the photon spectra of the observed VHE emission from AGN are often soft $\left(\Gamma_{o b s} \sim 3-5\right)$, and rarely is any emission observed above $\sim 1 \mathrm{TeV}$. This is in part due to the softening of the emitted blazar spectra via EBL effects, and is in part due to the VHE band often being located above the high-energy peak of the typical double-humped spectral energy distribution (SED) seen from AGN. The VHE flux observed from an AGN is almost always variable, similar to its behavior at other wavelengths. Typically variations of a factor of 2-3 are seen on timescales ranging from days to years. In other cases, particularly for non-HBL blazars, these variations are what temporarily made the object detectable at VHE. The detection of rapid (minute-scale), large-scale (factor of 100) variations of the VHE flux remains relatively rare.

Understanding VHE AGN and their related science relies on making precision measurements of their spectra, their variability patterns, and on contemporaneous multi-wavelength (MWL) observations. Typically these studies enable modeling of the AGN SEDs, as well as searches for correlations in the flux/spectral changes observed that may indicate commonalities in the origin of the observed emission. As third-generation VHE facilities such as VERITAS have been operating for nearly a decade, many of the major breakthroughs in research related to VHE AGN are now driven by the observation of major flaring episodes. Correspondingly, the VERITAS AGN program is now largely focused on the search for, and the observation of, these flares.

\section{VERITAS AGN Program}

VERITAS [1] is most sensitive between $\sim 85 \mathrm{GeV}$ and $\sim 30 \mathrm{TeV}$ and began routine scientific observations with the full array in September 2007. VERITAS can perform spectral reconstruction above $\sim 100 \mathrm{GeV}$, and has a sensitivity yielding a 5 standard deviation $(\sigma)$ detection of an object with flux equal to $1 \%$ Crab Nebula flux (1\% Crab) in $\sim 25$ hours. In Summer 2012, a major multi-step upgrade of VERITAS was completed, significantly improving its low-energy response. VERITAS now has $\sim 40 \%$ lower energy threshold, and detects AGN $\sim 2$ times faster than in 2009.

VERITAS acquires $\sim 980 \mathrm{~h}$ of good-weather observations each year during "dark time", and observations of AGN average $\sim 425 \mathrm{~h}$ per year. Historically, these data are split $\sim 90 \%$ to blazars, primarily BL Lac objects, and $\sim 10 \%$ to radio-galaxies, primarily M 87. Beginning in September 
2012, the VERITAS collaboration developed the capability to observed during periods of "bright" moonlight (i.e. $>30 \%$ illumination). These higher-threshold data add another $\sim 30 \%$ to overall good-weather data yield, of which $\sim 200 \mathrm{~h}$ per year are used for AGN studies. AGN comprise $63 \%$ of the VERITAS source catalog and Table 1 shows the 34 AGN detected by VERITAS.

Target-of-opportunity (ToO) observations have always been, and continue to be, a key component $(\sim 30 \%)$ of the VERITAS AGN (radio galaxy and blazar) program. However, the direction of the overall program has evolved over the years. In general, the philosophy has shifted from an emphasis on expanding the source catalog by discovering new VHE AGN, to exploiting the existing catalog via deep / timely measurements of the known sources. Initially the blazar program was $\sim 80 \%$ (2007-10) devoted to discovery efforts and following up on any successes. This effort is now $<20 \%$ of the AGN dark-time program (2012-15). Similarly the radio-galaxy program was $\sim 40 \%$ VHE discovery efforts, and no discovery data were taken the past three seasons. The VERITAS AGN program is now heavily devoted to regular VHE monitoring of the entire Northern VHE catalog, with a cadence designed to generate deep exposures for some particularly interesting targets, with intense MWL ToO follow-up planned for any interesting flaring events. These monitoring observations are coordinated with partners at lower energy, so that long-term contemporaneous MWL data sets exist for all Northern VHE AGN.

\section{Highlights from Blazar Discovery Observations}

Although the VERITAS VHE blazar discovery program has ramped down, there are a few recent highlights from it. These include the discovery of VHE emission from two blazars during ToO observations, and the preparation of limits for a sample of 114 blazars and 2FGL objects.

RGB J2243+203 is a Fermi-LAT-detected BL Lac object with unknown redshift. It has been classified as an IBL and an HBL, and is probably a borderline case. The favorable extrapolation of its hard LAT spectrum $\left(\Gamma_{2 F G L} \sim 1.75, \Gamma_{1 F H L} \sim 2.4\right)$ motivated $\sim 5 \mathrm{~h}$ of observation of this object by VERITAS in 2009, but it was not detected, yielding a flux limit of $\sim 2 \%$ Crab. Although previous observations were unsuccessful, this object was one of hundreds of candidate VHE emitters whose Fermi-LAT flux and photon index is automatically monitored on timescales ranging from $0.25-28$ days by VERITAS. On December 20, 2014, this pipeline issued a flaring alert based on a relative flux change (important for HBLs) which initiated VERITAS ToO observations. It is notable that the triggering flux was below the threshold typically used by the Fermi-LAT team for alerts. The VERITAS observations yielded $\sim 4 \mathrm{~h}$ of good-quality data over the following four nights, which were effectively the end of the observing season for the target. An excess of $\sim 200 \gamma$-rays $(\sim 6 \sigma)$ was detected from RGB J2243+203. The observed flux above $160 \mathrm{GeV}$ is $\sim 6 \% \mathrm{Crab}$ and the observed photon index is $\Gamma=4.6 \pm 0.6$. More details on these studies are presented in [3].

S3 1227+25 (ON 246) is a Fermi-LAT-detected IBL at a redshift of $z=0.135$. Its MeV-GeV properties $\left(\Gamma_{3 F G L} \sim 2.2, \Gamma_{1 F H L} \sim 3.3\right)$ suggest it is an unlikely VHE emitter during its baseline emission state. In January 2015, the object flared initiating alerts from both the LAT team and the automated VERITAS pipeline. In response, VERITAS acquired $\sim 3 \mathrm{~h}$ of good-weather ToO observations, but did not detect the source despite the reports of $\sim 30$ times brighter MeV-GeV flux $\left(\Gamma_{L A T}=2.2\right)$. In May 2015, an even brighter flare $\left(\sim 40 \mathrm{x}\right.$ flux, $\left.\Gamma_{L A T}=1.9\right)$ of this blazar initiated similar alerts, triggering another VERITAS campaign lasting about one week. Overall, an excess of $420 \gamma$-rays $(\sim 13 \sigma)$ was detected from S3 1227+25. Figure 1 shows the sky map of the significance 


\begin{tabular}{|c|c|c|c|}
\hline AGN & $z$ & Type & $\log _{10}\left(v_{\text {synch }}\right)[\mathrm{Hz}]$ \\
\hline M 87 & 0.004 & FR I & -- \\
\hline NGC 1275 & 0.018 & FR I & -- \\
\hline Mrk 421 & 0.030 & HBL & 18.5 \\
\hline Mrk 501 & 0.034 & HBL & 16.8 \\
\hline 1ES 2344+514 & 0.044 & HBL & 16.4 \\
\hline 1ES 1959+650 & 0.047 & HBL & 18.0 \\
\hline 1ES 1727+502 & 0.055 & HBL & 17.4 \\
\hline BL Lac & 0.069 & IBL & 14.3 \\
\hline 1ES 1741+196 & 0.084 & HBL & 17.9 \\
\hline W Comae ${ }^{\dagger}$ & 0.102 & IBL & 14.8 \\
\hline RGB J0521.8+2112 ${ }^{\dagger}$ & 0.108 & HBL & -- \\
\hline RGB J0710+591 ${ }^{\dagger}$ & 0.125 & HBL & 21.1 \\
\hline H 1426+428 & 0.129 & HBL & 18.6 \\
\hline $\mathrm{S} 31227+25^{\dagger}$ & 0.135 & IBL & 14.8 \\
\hline 1ES $0806+524^{\dagger}$ & 0.138 & HBL & 16.6 \\
\hline 1ES $0229+200$ & 0.140 & HBL & 19.5 \\
\hline 1ES $1440+122^{\dagger}$ & 0.162 & IBL & 16.5 \\
\hline RX J0648.7+1516 & 0.179 & HBL & -- \\
\hline 1ES 1218+304 & 0.184 & HBL & 19.1 \\
\hline RBS $0413^{\dagger}$ & 0.190 & HBL & 17.0 \\
\hline 1ES 1011+496 & 0.212 & HBL & 16.7 \\
\hline MS $1221.8+2452$ & 0.218 & HBL & 14.0 \\
\hline 1ES 0414+009 & 0.287 & HBL & 20.7 \\
\hline $3 \mathrm{C} 66 \mathrm{~A}^{\dagger}$ & $0.33<z<0.41$ & IBL & 15.6 \\
\hline PKS 1222+216 & 0.432 & FSRQ & -- \\
\hline PG 1553+113 & $0.43<z<0.50$ & HBL & 16.5 \\
\hline PKS $1424+240^{\dagger}$ & $z>0.604$ & IBL & 15.7 \\
\hline PKS 1441+25 & 0.939 & FSRQ & -- \\
\hline 1ES $0033+595$ & $?$ & HBL & 18.9 \\
\hline $1 \mathrm{ES} 0502+675^{\dagger}$ & $?$ & HBL & 19.2 \\
\hline 1ES $0647+250$ & $?$ & HBL & 18.3 \\
\hline B2 1215+30 & $?$ & IBL & 15.6 \\
\hline HESS J1943+213 & $?$ & HBL & -- \\
\hline RGB J2243+203 ${ }^{\dagger}$ & $?$ & HBL & 14.2 \\
\hline
\end{tabular}

Table 1: The 34 AGN (25 HBL, 7 IBL, 2 radio galaxies) detected with VERITAS. This catalog has grown by 7, 13, and 22 AGN since the ICRCs in 2013, 2011 and 2009, respectively. The 12 blazars discovered at VHE by VERITAS are marked with a dagger. The classifications and synchrotron peak frequencies are taken from TeVCat and [2], respectively.

for the region surrounding S3 $1227+25$. The blazar is clearly detected on three separate nights: May 16, 18, and 21, 2015 (UTC), noting that weather issues affected the temporal coverage. A variable, soft-spectrum flux was observed, with peaks of 6-8\% Crab above $100 \mathrm{GeV}$.

Upper Limits were derived from VERITAS observations (2007-2012) of 93 blazars that were not eventually detected as VHE emitters. The observations of these objects comprise a total goodquality live time of $\sim 570$ hours. While none of these individual sources were detected, a $4.6 \sigma$ (pre-trials) excess is seen if one stacks the observations of all 36 relatively nearby $(z<0.6)$ HBLs in the sample, i.e. the dominant population of extra-galactic VHE sources. No significant excess is seen $(0.6 \sigma)$ by stacking the remaining targets. Figure 1 shows the distribution of integral flux upper limits derived from VERITAS observations of each of these blazars, as well as for the 21 2FGL sources serendipitously located in the $3.5^{\circ}$ field of view of VERITAS. The typical limit from this sample is $\sim 2 \%$ Crab, and often the most sensitive produced at VHE. 

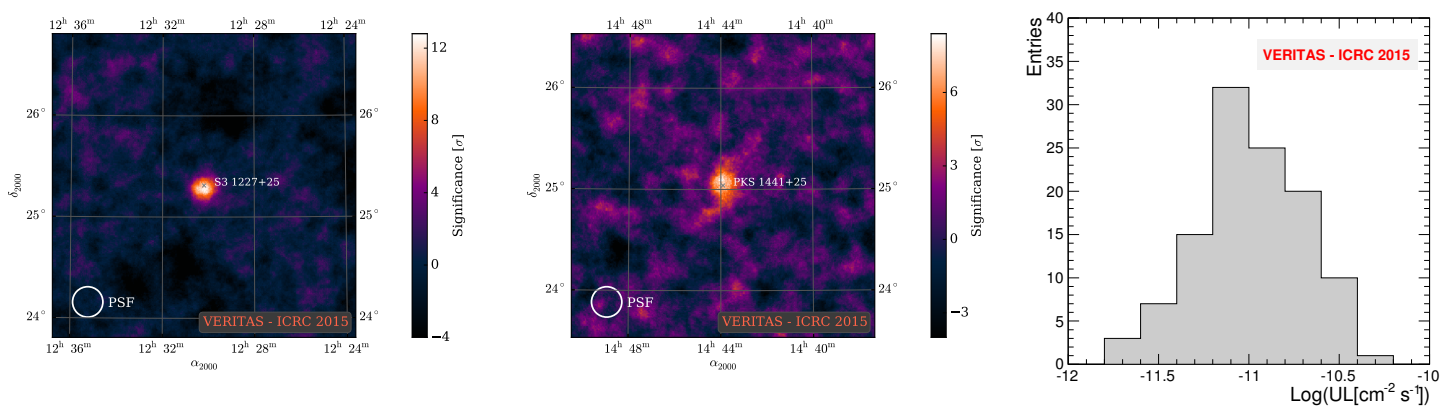

Figure 1: The preliminary sky maps of the significance measured from the direction of S3 1227+25 (left) and PKS $1441+25$ (center). Right) The preliminary histogram of integral flux upper limits derived from VERITAS observations of 93 blazars and all 21 other 2FGL sources in the field of view of those blazars.

\section{Highlights from Observations of Known VHE Blazars}

Observations of known VHE blazars are the dominant component of the VERITAS AGN program. These data include various long-term flux monitoring programs, ToO observations and single-season campaigns. A few recent highlights are given here, as well as some results that have further guided the development of the VERITAS blazar observing program.

PKS 1441 +25 is an FSRQ located at a redshift of $z=0.939$. Immediately after the MAGIC discovery (ATel \#7402) of VHE $\gamma$-ray emission from this object in April 2015, VERITAS initiated a ToO observation campaign. A total of $15 \mathrm{~h}$ of good-quality observations was acquired during a 1 -week period, resulting in the detection of a very soft spectrum excess of $\sim 400$ events $(\sim 8 \sigma$; see Figure 1). The observed flux was steady at $\sim 5 \%$ Crab above $80 \mathrm{GeV}$. A further $\sim 4 \mathrm{~h}$ of data were taken in May 2015, after the MeV-GeV (Fermi-LAT) flare that initiated the MAGIC observations had subsided. No significant excess was observed from the source, unlike the VERITAS detection ( $\sim 6 \sigma$ in $\sim 6$ h over 10 nights) of steady emission ( $\sim 3 \% \mathrm{Crab}$ ) from the FSRQ PKS 1222+216 approximately two weeks after a similar MeV-GeV flare had subsided in February 2014.

1ES 0229+200 was observed by VERITAS for 54.3 h from 2009-2012 as part of a MWL monitoring campaign including Swift, RXTE and Fermi-LAT [4]. The blazar was solidly detected by VERITAS $(\sim 12 \sigma)$ and the VHE spectrum well measured $(\Gamma=2.59 \pm 0.12)$. A variable VHE flux was observed presenting challenges to efforts to exploit the VHE spectrum of 1ES 0229+200 to constrain the strength of the intergalactic magnetic field. In addition, a synchrotron self-Compton (SSC) model was fit to the SED. Rather than presenting a single, degenerate solution the entire range of SSC parameters allowed by the data were presented. Although no detection was particularly strong in any given waveband, in many cases the SSC parameters were constrained to a factor of $\sim 2$ showing the general promise for VERITAS MWL campaigns on other blazars.

PKS 1424+240 is an IBL discovered at VHE by VERITAS in 2009 [5]. In 2013, archival HST measurements were used to show it has $z>0.604$, making it one of the most distant VHE blazars [6]. This motivated a deep campaign on this object in 2013 [7]. In total more than $100 \mathrm{~h}$ of observations, including archival data, resulted in a $\sim 18 \sigma$ detection above $120 \mathrm{GeV}$ and a VHE spectrum that is now well measured $(\Gamma=4.2 \pm 0.3)$. When EBL effects are removed assuming the redshift is at the lower limit, the spectrum shows an indication of spectral hardening that is curious and becomes more so with higher redshift assumptions. Unfortunately the VHE flux was more 
than 2 times lower in 2013 than in 2009, and has remained low since, thus an improvement in the high-energy statistics further probing this unusual spectral signature has remained difficult.

HESS J1943+213 is a point-like, $>500 \mathrm{GeV}$ emitter found in the HESS Galactic Plane scan [8]. It is a hard-spectrum Fermi-LAT source and might be a blazar, but no flux variations have been observed, nor has any redshift been measured. To probe the object's possible blazar origins, VERITAS observed HESS J1943+213 for $22.5 \mathrm{~h}$ of good-quality live time in 2014. A strong excess $\left(18 \sigma ; 3.7 \sigma \mathrm{h}^{-0.5}\right)$ was observed, corresponding to a flux that is consistent with the HESS (200508) measurement. No flux or spectral variations are seen on daily or weekly time scales. The observed spectrum $(\Gamma=2.82 \pm 0.13)$ is harder than, albeit consistent with, the HESS spectrum $(\Gamma=3.1 \pm 0.3)$, whose softness was a key motivator for the blazar interpretation of this VHE source. More details on these VERITAS studies can be found in [9].

1ES 1727+502 was detected by VERITAS for the first time during a flare in May 2013 [10]. The observed peak integral flux $(\sim 10 \% \mathrm{Crab})$ is about five times higher than the archival VHE flux. The detection is notable since it was achieved using observations with a reduced-high-voltage configuration that enables observations under bright moonlight. This detection led to the targets of the VERITAS bright moonlight program being expanded from a few, generally bright, hard-VHEspectrum blazars to a wide variety of targets, including VHE discovery candidates.

A Snapshot Program was developed by the VERITAS collaboration on a trial basis during the 2013-14 season. Here, a 15-minute observation was taken each month during the four best months of visibility for each of $\sim 20$ VHE blazars not otherwise studied or monitored by VERITAS. This snapshot enables VERITAS to detect an excess from a $>10 \%$ Crab source, which in most cases would correspond to a bright VHE flare from the object. While this program would likely miss any short-duration flaring events (e.g. $<1$-day flares), the hope was that it would likely catch many longer-duration events that could be followed up with coordinated MWL campaigns. This program proved immensely successful. During the 2013-14 season, five flaring blazars were detected by the snapshot program, including 1ES 0033+595 ( 20\%, 15\% Crab), 1ES 1011+496 (peaking at $\sim 70 \% \mathrm{Crab}$ ), MS 1221.8+2452 ( 15\% Crab), VER J0521+211 (peaking at $\sim 40 \%$ Crab; see [11]), and 1ES 1727+502 ( 10\% Crab). These flares complemented other flares found via VERITAS' intense blazar monitoring programs (e.g. a 1-day VHE 15\% Crab flare of BL Lac in 2013; a $>200 \%$ Crab flare of B2 1215+30 - see [12]), or via ToO observations triggered by events at lower energy (e.g. PKS $1222+216$ in 2014). Following up on this trial, a more intense Snapshot Program was carried out in 2014-15 (see below). Although the results were less spectacular, elevated states were found from several of the targets.

\section{Long-term Blazar Observing Strategy}

In Fall 2014, a new long-term (5-year) strategy for the VERITAS AGN observation program during dark-time was implemented. While the program will naturally evolve, the following describes the current strategy. For blazars, deep observations via intense, regular monitoring will be taken for 10 blazars fitting into three core programs (128 h total). One core program will focus on EBL and Cosmic-ray Line of Sight Measurements via observations of five moderately distant, hard VHE spectrum blazars (1ES 0229+200, RGB J0710+591, 1ES 1218+304, PKS 1424+240 and H 1426+428). Another core program will focus on Understanding MWL Variations via observations of three blazars that are highly variable at all wavelengths (3C 66A, W Comae and BL Lac), 


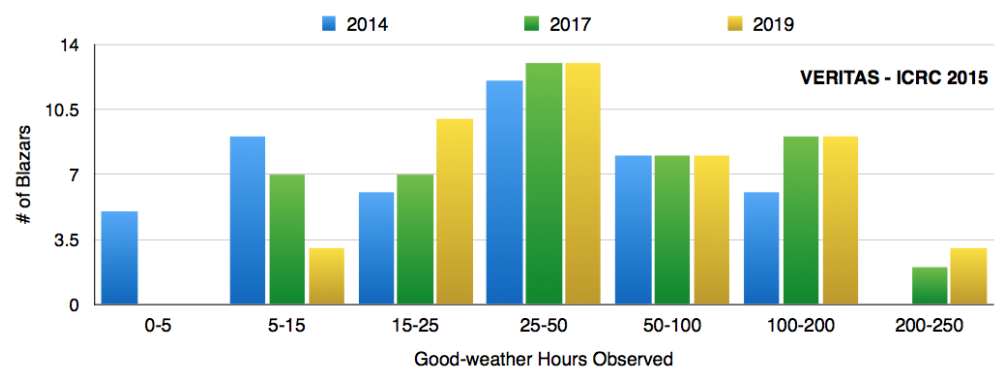

Figure 2: The histogram of the actual, or minimally expected, VERITAS exposure for each Northern Hemisphere blazar in July of 2014 (mean $=32 \mathrm{~h}$; median $=46$ h), 2017 (mean $=39$ h; median = 61 h) and 2019 ( mean = 44 h; median $=71 \mathrm{~h}$ ). No observations beyond the monitoring programs (e.g. ToO data) are assumed. Three blazars not known to be VHE emitters in July 2014 are included.

Another core program will focus on Iconic Objects by generating regular single-night spectra for Mkn 421 and Mkn 421, as components of a major MWL effort. Another key component of the long-term strategy is the Snapshot Program. Here, VERITAS will take weekly snapshot observations of any other visible targets remaining in the Northern VHE blazar catalog (36 targets), to detect flaring events (program total $=86 \mathrm{~h}$ ). While the duration of each target's snapshot varies (typically $15 \mathrm{~min}$, up to $1 \mathrm{~h}$ ), the minimal detection sensitivity of any snapshot is $10 \%$ Crab flux. An automatic, real-time analysis pipeline and a comprehensive decision tree for ToO-triggering ensures that instantaneous follow-up of any flare at least five times the base-line flux (minimally $10 \% \mathrm{Crab}$ ) occurs. Each year an 80 -h allocation is pre-approved for $\mathrm{ToO}$ follow-up of flaring events meeting any of a number of MWL / VHE triggers; the pre-approval exists to reduce logistical hurdles for triggering, including instantaneous ones, and additional ToO time is possible. The aforementioned monitoring observations are taken simultaneously with Swift UVOT / XRT for the 10 core-program blazars, as well as for 7 other high-priority blazars. For each of the 46 blazars intense BVri coverage is arranged with the FLWO 48-inch optical telescope. Additional time $(\sim 100$ $\mathrm{h} /$ year) is envisioned for Other Blazar Projects.

Overall, this program should provide regular sampling of the light curves of all NorthernHemisphere VHE blazars for a five year period, and in 15 cases for a $\sim 10$-year period, all with intense MWL coverage. In addition, every VHE blazar visible to VERITAS will eventually have reasonably deep coverage by 2019. Figure 2 shows a histogram of the minimum total VERITAS exposure for each of the Northern-Hemisphere VHE blazars that will be acquired under this plan by July 2017 and July 2019, as well as where the exposures stood in July 2014.

\section{Recent Radio Galaxy Studies \& Future Plans}

The VERITAS radio-galaxy program has long consisted of deep monitoring of the known VHE emitter M 87, ToO observations on the known VHE emitter NGC 1275 (since 2009), and discovery observations of a few EGRET- and Fermi-LAT-detected objects. Recently the discovery component was converted to a Fermi-LAT-based ToO program. The M 87 program has been very successful, with $\sim 230 \mathrm{~h}$ of good-weather data being acquired, along with intense MWL coverage and the observation of bright flares in 2008 [13] and 2010 [14]. Recently the AGN has been fairly quiet aside from a relatively minor brightening in 2012 [15]. In the past two seasons, the target was only weakly detected $(\sim 6 \sigma)$ in modest exposures $(<16 \mathrm{~h}$ good-quality live time) taken each season, with 
the observed flux near the baseline value $(\sim 1.5 \% \mathrm{Crab})$. NGC 1275 was recently detected $(\sim 7 \sigma)$ by VERITAS during ToO observations taken in December 2012 - February $2013(\sim 16 \mathrm{~h})$ and in October - November $2013(\sim 16 \mathrm{~h})$. A soft-spectrum $(\Gamma \sim 4 ; 1 \%$ Crab) was observed, consistent with prior MAGIC measurements. In the future, M 87 will continue to be regularly monitored (20 $\mathrm{h} / \mathrm{yr}$ ) as part of a major world-wide effort, and ToO observations will be taken of NGC 1275, as well as any Fermi-LAT-detected radio galaxy should the LAT flux exceed a prescribed threshold.

\section{Conclusions}

AGN observations remain a major component $(\sim 50 \%)$ of the scientific program of VERITAS. Thirty-two BL Lac objects, two FSRQs and two FR-I radio galaxies are detected with the observatory, and constraining upper limits exist from VERITAS observations of $\sim 100$ other AGN. A strategy guiding the VERITAS AGN program through 2019 is organized. This strategy is heavily focused on regular VHE and MWL monitoring of all known VHE AGN in the Northern Hemisphere, and intense ToO follow-up of interesting flaring events. Although the VERITAS AGN discovery program has decreased in scope, the collaboration continues this effort, largely with ToO observations during "dark time" and non-ToO bright-moon observations. Given the major commitment to AGN research, there should be many exciting VERITAS results still to come.

This research is supported by grants from the U.S. Department of Energy Office of Science, the U.S. National Science Foundation and the Smithsonian Institution, and by NSERC in Canada. We acknowledge the excellent work of the technical support staff at the Fred Lawrence Whipple Observatory and at the collaborating institutions in the construction and operation of the instrument. The VERITAS Collaboration is grateful to Trevor Weekes for his seminal contributions and leadership in the field of VHE gamma-ray astrophysics, which made this study possible.

\section{References}

[1] http://veritas.sao.arizona.edu/specifications

[2] E. Nieppola et al., SEDs of a Large Sample of BL Lacertae Objects, A\&A, 445, 441, 2006.

[3] U. Abeysekara, VERITAS Discovery of VHE $\gamma$-Ray Emission from RGB J2243+203, these proceedings, 2015.

[4] E. Aliu et al., A Three-Year MWL Study of the VHE gamma-ray Blazar 1ES 0229+200, ApJ, 782, 13, 2014.

[5] V. Acciari et al., Discovery of VHE $\gamma$-rays from PKS 1424+240 \& MWL constraints on its redshift, ApJ, 708, L100, 2010.

[6] A. Furniss et al., The Firm Redshift Lower Limit of the Most Distant TeV-detected Blazar PKS 1424+240, ApJ, 768, L31, 2013.

[7] S. Archambault et al., Deep Broadband Observations of the Distant Gamma-ray Blazar PKS 1424+240, ApJ, 785, L16, 2014.

[8] A. Abramowski et al., HESS J1943+213: a candidate extreme BL Lacertae object, A\&A, 529, 10, 2011.

[9] K. Shahinyan, VERITAS Observations of HESS J1943+213, these proceedings, 2015.

[10] S. Archambault et al., VERITAS Detection of $\gamma$-ray Flaring Activity from the BL Lac Object 1ES 1727+502 During Bright Moonlight Observations, ApJ, in press, 2015 [arXiV:1506.06246].

[11] H. Prokoph, Time-resolved multiwavelength observations of the blazar VER J0521+211 from radio to gamma-ray energies, these proceedings, 2015.

[12] F. Zefi, A bright $\gamma$-ray flare from the blazar B2 1215+30 detected by VERITAS and Fermi-LAT, these proceedings, 2015.

[13] V. Acciari et al., Radio imaging of the VHE $\gamma$-ray emission region in the central engine of a radio galaxy, Science, 325, 444, 2009.

[14] E. Aliu et al., VERITAS observations of day-scale flaring of M87 in April 2010, ApJ, 746, 141, 2012.

[15] M. Beilicke, VERITAS observations of M87 in 2011/2012, AIPC, 1505, 586, 2012. 